\title{
Modern surgery and ancestor reflux
}

\author{
F. M. Riegler
}

Published online: 7 October 2016

C Springer-Verlag Wien 2016

Dear reader,

Welcome to the present issue of European Surgery, including fascinating reports on vascular, hepatobiliary, endocrine, general and basic science surgery. In addition, a behind the scenes look at the process of reasoning is given in a paper that analyses the medical and surgical background of northern mythology.

First of all, northern mythology (Saga, Edda) remembers the roots of the European culture. As such, myths aim to express deep knowledge and legacy from our ancestors. However, problems usually arise as modern man has difficulty in understanding the deep content and meaning of myths. The language is old fashioned and myths operate with specific pictures, images and symbolisms, the meanings of which have been lost and forgotten (i.e. scientific, astronomical knowledge). It remains to be questioned whether myths may be of interest for modern medicine at all?

Conceptually, a patient history describes an individual story and the patient uses his own words and images to describe his or her condition. The deep meaning of the patient history guides the way straight towards diagnosis and treatment. As such, patients frequently report which conditions contribute to attenuate the severity of symptoms and signs. Furthermore, symbolisms used to list causative situations mirror the language of myths. Thus, the patient history may be seen as a special form of myth and thus everybody may be interpreted as a walking rhythm \& blues, reggae or soul song, opera or symphony. It remains to be questioned how much legacies may be of importance.

\section{F. M. Riegler $(\bowtie)$}

Reflux Medical, Mariannengassse 10/9, 1090 Wien, Austria martin.riegler@refluxmedical.com
Let us turn the wheel and ask ourselves to what extent, in addition to presenting a disease measurable by all the fascinating tools of modern medicine, signs \& symptoms may have a deeper meaning. May patient histories contain information comparable to an individual myth? May stories of patients mirror a deep layer of information, which is usually neglected, since we seem to have lost the sensitivity for such streams of emotion and lack adequate time to listen?

Ectoderm- (nerve \& epithelial cell-) based manifestations, including inflammatory oesophageal disease (eosinophilic esophagitis; gastroesophageal reflux disease, GERD), achalasia, dyspepsia, irritable bowel disease, inflammatory bowel disease, neurodermatitis, psoriasis, migraine, hypertension and many more share a common characteristic of the patient history of those affected. Some of the themes and melodies of their blues are related to "ancestor reflux". Psychosomatic medicine lists a huge number of ancestor reflux conditions as being essential for development of the above diseases, including imbalance regarding the relationship to parents, brothers and sisters. Such ancestor reflux may also affect entire civilizations, religious and political systems. Our world knows plenty of it. In contrast to this, music does not know racism, religion, politics, hate, greed, envy and war. However, man learned to misuse music for the abovementioned justifications of power games. Flood myths contain the best known stories on global ancestor reflux. Using mythological semiotics, these legacies describe the idea of a lost civilization, which was extinguished 10,000-9000 years B.C. In line with recent investigations, the end of this culture may be related to a global cataclysm induced by several cosmic impacts. The manifestations of the impacts are described in the flood myths all over the world and list thunderstorms, floods, global winter and longstand- 

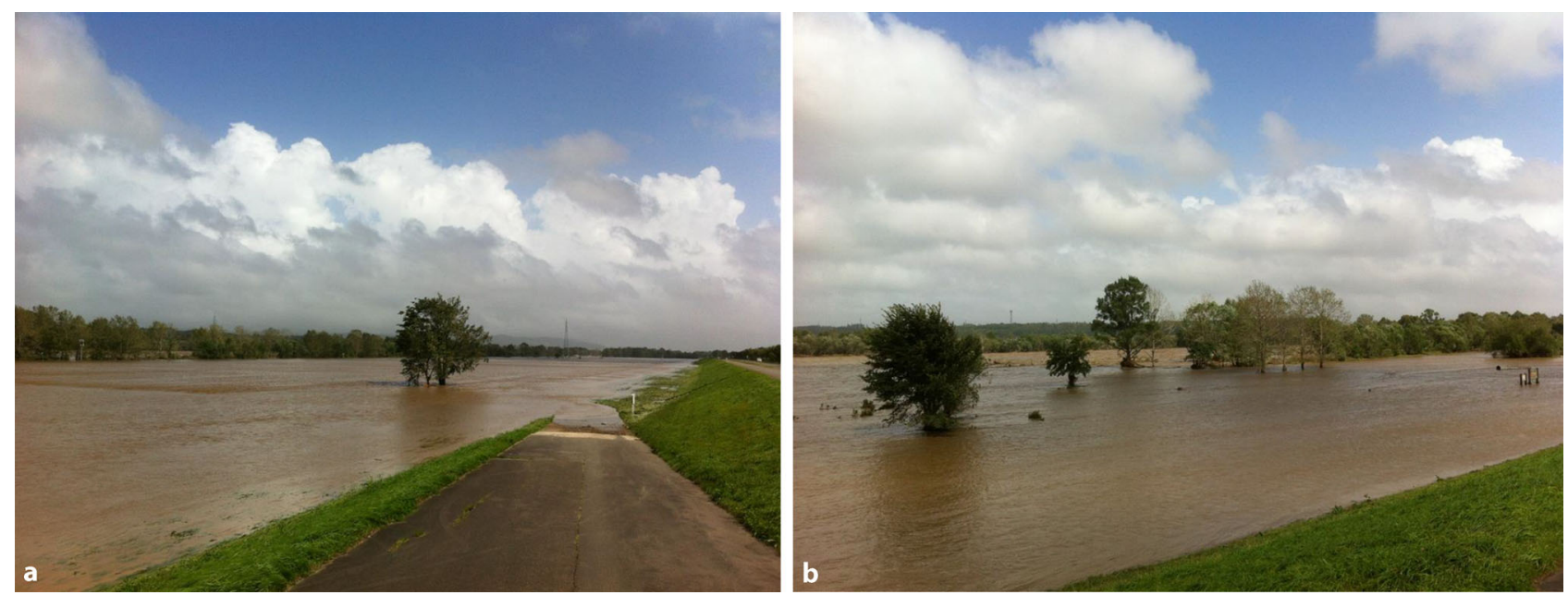

Fig. 1 Imagesa and b show the flooding following a typhoon, which hit the Japanese Island of Hokkaido end of August2016. Images aim to mirror the idea of the author regarding the semiotics of ancient flood tales, as outlined in the text

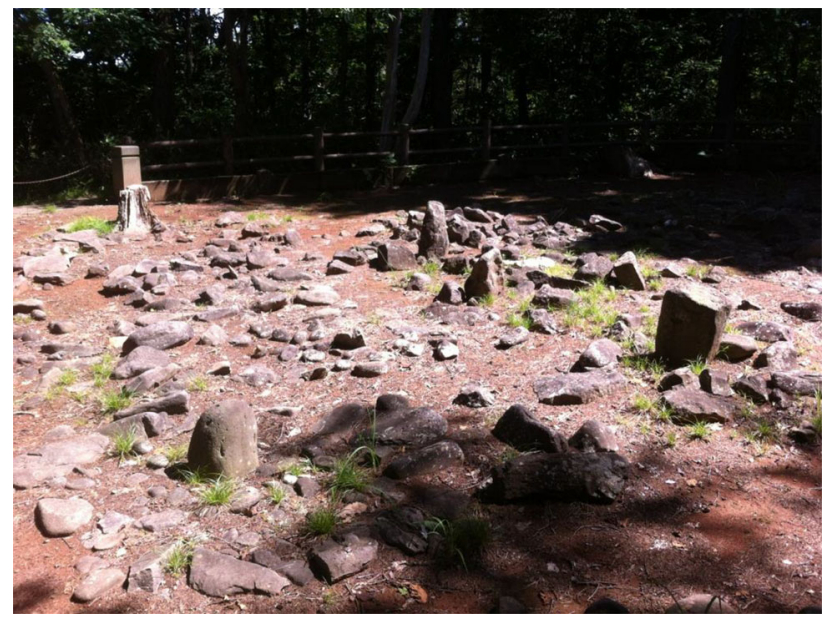

Fig. 2 Stone Age circles of the Jomon culture, image obtained at Hokkaido, Japan. The circles are oriented according to stellar positions. Age of the man-made structures is approximately 5000-6000 years before present. Astronomical knowledge contained within the structures may originate from a know-how transfer going back to the pre-flood culture, as outlined in the text

ing darkness (Fig. 1a and b). In line with the flood myths, the ice-core analysis of the Greenland ice sheet revealed a significant temperature decline during this particular time period, also known as the "young dryas". According to the numerous publications by Graham Hancock, Anthony West, Robert Bauval and Robert M. Schoch, it is suggested that the survivors of this cataclysm set the basis for the development of precursors of our present civilization (Fig. 2 and 3). Thus, the legacy of this pre-flood survived in the stories surrounding Atlantis and the mythologies of the ancient cultures (Egypt; Mesopotamia; North, Central and South America; Middle and Far East; Pacific and Australia). It remains to be questioned whether remnants of pre-flood culture and architecture exist, i. e. before $9000-10,000$ years B.C.
The temples of Göbekli Tepe in south-eastern Turkey were abandoned around 9600 B.C., i.e. the period when "the stars fell from heaven", and Plato dated the extinction of Atlantis. Subterranean multifloor cities and tunnel systems have been excavated in Turkery, Iran, Egypt and South America, the youngest parts of which are dated within the period between 9000 and 10,000 years B.C. Most fascinating similar tunnel systems have now been found by Heinrich Kusch in Austria (i. e. Vorau in Eastern Styria, Klosterneuburg in Lower Austria). According to novel technologies, which allow the age of the tunnels to be dated, the older parts of these man-made structures in Austria are suggested to have been created between 20,000 and 30,000 years ago. In line with these data, remnants of animals that became extinct around 14,000-20,000 years ago have been found within these tunnels. Taken together, these data indicate that our understanding of history should be reconsidered. Finally, it remains to be questioned what impact these data have on the idea that patient history mirrors an individual myth.

May it be that there is a deeper meaning of a disease that cannot be directly translated into measurable signs? May it be that disease aims to mirror a lack of wellbeing due to a deficit of adequate discipline (nutrition, life style), respect and attention (family, business)? Do symptoms contain a deeper layer of semiotics, such as: "My symptoms should be taken more seriously by the physician?" May the images used by the patients to describe their state of mood, including, "I cannot swallow that any more", "It causes heartburn", "Whenever I remember a particular situation of my life (i. e. death of father, mother, kid etc.)", "It tends to explode my brain" etc., simply be nothing other than mythological signs for ancestor reflux, at least in part? It seems that modern medicine should address this topic, and include it in the diagnostic and therapeutic algorithm. Modern surgery treats nu- 

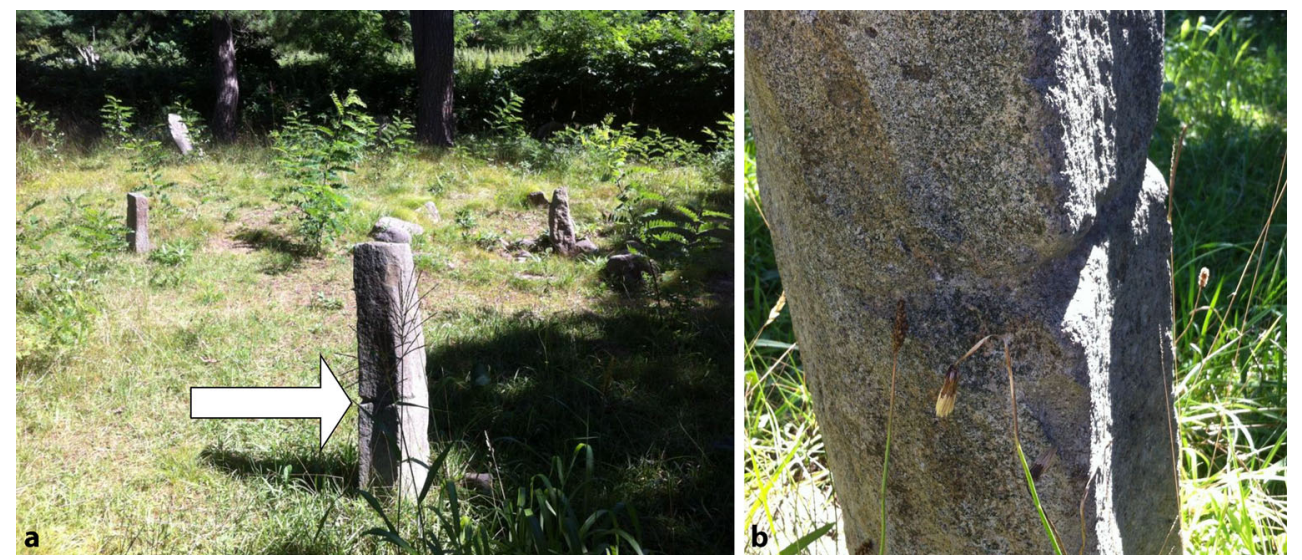

Fig. 3 Stone Age circle of the Jomon culture (Hokkaido, Japan) arrangement is based on ancient astronomical knowledge, as outlined in the ext. Arrow in a marks stone incision which is depicted in larger scale in $\mathbf{b}$. Note that the incision in the stone aimed to serve to target the position of the sun and stars during the solar year (solstices, equinoxes). Thus, the stone circle served as a calendar, the prerequisite for agriculture and civilization. Age of the stone circle is around 5000-6000 years before present

merous functional and oncological diseases for which it has been demonstrated that psychological themes are of major importance. Finally, the possible impact of the above observations for our modern civilization may be questioned.

It seems that we should optimize or at least improve our style of communication with each other, reduce the powers of hate, envy, greed and jealousy in favour of gratitude, respect and humility. Then we would profit from the ancient myths and avoid repeating the faults of previous cultures. Be yourself and harmonize your ancestor reflux; stay tuned and well balanced.

Acknowledgements The author thanks his patients, from whom he got the idea that medical histories contain a deeper, mythological message that may tell essential truths about our own present civilization. Furthermore, the author thanks his family and friends for major support, passion and motivation.

Conflict of interest F.M. Riegler declares that he has no competing interests. 